\title{
Contexte territorial et organisation scolaire: l'école rurale française, de l'idéalisation à la stigmatisation.
}

\section{Yves Alpe}

Le système éducatif en milieu rural est depuis son origine traversé par des tensions aggravées par l'évolution récente du contexte socio-économique du monde rural. La fonction de socialisation de l'école rurale a souvent été mise en avant, mais les performances scolaires des élèves ruraux, supposées inférieures à celles des urbains, ont fait l'objet de nombreuses études, qui posent d'une part la question de la stigmatisation des territoires ruraux et de ses effets sur les acteurs scolaires, et d'autre part celle des politiques éducatives territorialisées, souvent en décalage avec les résultats de la recherche, comme le montre l'exemple de la France développé ici.

\section{Introduction}

Les systèmes éducatifs se donnent presque partout une vocation universelle, ou même universalisante: l'école doit s'adresser à tous les enfants, sans discrimination liée à des contextes socioculturels et économiques. Si cela était vraiment le cas, la question de «l'école rurale» (comme celle d'ailleurs de «l'école des banlieues» ou de «l'école urbaine») n'aurait pas de sens. Le fait qu'elle soit largement évoquée, qu'elle ait fait l'objet de nombreux travaux de recherche dans de nombreux pays, qu'elle fasse l'objet de politiques éducatives dites "territorialisées», montre que l'hypothèse dominante est plutôt celle de l'existence de spécificités liées à des effets de contexte, et tout particulièrement des "effets de territoire». Cet article analyse le cas de la France, en le positionnant dans la première partie dans un contexte plus large à travers quelques ouvertures sur des exemples étrangers, principalement européens, et en précisant les grandes lignes de son évolution récente. La deuxième explore la question des "effets de territoire» à partir de résultats d'enquêtes, la troisième s'interroge sur la stigmatisation des territoires par les politiques publiques d'éducation et ses effets sur les acteurs scolaire. 


\section{Le système éducatif en zone rurale: des questionnements largement répandus}

\section{L'école rurale aujourd'hui}

La question de l'école rurale se pose dans un très grand nombre de pays, car les politiques éducatives ont toujours été confrontées à une réalité contraignante: l'inégale répartition de la population sur le territoire. Celle-ci acquiert une importance considérable lorsque l'on se donne comme objectif de mettre en place une "école pour tous», qui par définition doit offrir partout les mêmes contenus, à partir d'une même organisation systématique, la "forme scolaire» (Monjo, 1998; Vincent, 1994). En effet, les sociétés à forte dominante rurale connaissent un morcellement et une dispersion géographique des communautés qui rend difficile la concentration des populations scolaires, et donc l'organisation de l'offre scolaire: les écoles doivent être les plus nombreuses là où il y le moins d'habitants! Cette difficulté a été très tôt identifiée lors de la construction du système éducatif français, comme en témoigne la citation suivante:

Il y avait, au mois de mai 1899, 19724 élèves fréquentant les écoles, tant publiques que privées, du département [des Basses-Alpes], soit une proportion de 2,82 élèves par kilomètre carré. C'est là une condition géographique et économique exceptionnelle, qui force à multiplier les écoles au-delà des besoins réels, parce qu'on veut, et avec raison, éviter aux jeunes enfants des parcours longs et pénibles pour se rendre en classe et se procurer les bienfaits de l'instruction. (Dauthuile, 1900, p. 3)

Aujourd'hui, deux situations très contrastées peuvent être identifiées:

- Celui de sociétés encore largement rurales, à niveau de vie faible ou très faible, où le premier problème est de généraliser la scolarisation et l'éducation de base: les questions centrales sont - outre la faiblesse de moyens et des effectifs souvent énormes, ainsi près de 100 élèves par classe dans les zones rurales du Burkina Faso (Alpe \& Fauguet, 2008) - celles de la présence de l'école et de la régularité de la présence des élèves;

- Celui des sociétés développées à haut niveau de vie, dans lesquelles l'essentiel de la population est concentré dans les villes, où la question de l'école rurale s'exprime surtout par des préoccupations relatives à la qualité de l'enseignement.

Cette dichotomie existe en Europe: dans l'UE à 27, le rural représente 91\% du territoire, et seulement $56 \%$ de la population. Selon la classification OCDE, $40 \%$ de l'Europe est "principalement rurale» (Commission européenne, 2008, document non paginé). Mais les écarts sont énormes: la population rurale représente 2,7\% en Belgique, 14,1\% au Danemark, 24, 5\% en France, 42,4 \% au Portugal et $50,5 \%$ en Slovénie. De ce fait, comme le souligne la Commission européenne, «toute définition de la ruralité se ressent d'un certain degré d'arbi- 
traire dans le choix des seuils des variables. Face au contexte européen, cet arbitraire augmente à cause de la grande hétérogénéité des zones rurales» (2008, document non paginé). Les définitions de la ruralité sont variables, mais dans tous les cas elles sont d'abord fondées sur des indicateurs relatifs à la population (nombre d'habitants regroupés dans une unité de base, comme les "communes» qui ont servi de base à l'analyse en France, ou densité de la population), parfois accompagnés d'indicateurs socioéconomiques (activité économique dominante, part de l'emploi agricole dans l'emploi total, rapport entre le nombre d'actifs et le nombre d'emplois dans l'unité de base,...).

Il existe aussi des points communs: la population rurale est globalement en baisse, et sa composition socio-économique se modifie très vite, avec l'arrivée de trois grandes vagues de "néo-ruraux», d'importance variable selon les régions: inactifs âgés (surtout dans le Sud de l'Europe), ménages instruits à haut niveau de vie et mobiles ayant fait le choix d'une qualité de vie, et populations en difficultés chassées de la ville par la crise économique. Aujourd'hui, les ruraux dépendent de moins en moins de l'agriculture, et l'hétérogénéité sociale des populations rurales s'accroît dans tous les pays, tandis que la demande sociale d'éducation s'homogénéise: celle des ruraux se rapproche, comme dans d'autres domaines culturels, de celle des urbains.

De plus, l'école rurale possède partout un ensemble de caractéristiques quasi identiques: petite taille des unités, classes multiâges, relations étroites avec la communauté locale. Et elle connaît partout, à des degrés divers, les mêmes problèmes: isolement géographique, faiblesse relative des ressources, disparition accélérée des plus petites structures et tendance marqué au regroupement de classes et d'écoles. Cela se vérifie au Québec (Carrier \& Beaulieu, 1995) comme en Catalogne (GIER, 2006), en Roumanie (Alpe, 2002) comme dans les départements ruraux français.

Dans de nombreux cas aussi, on peut identifier des déficits dans l'offre scolaire en milieu rural pour la pré-scolarisation: «En Pologne, par exemple, en 2003 le pourcentage d'enfants de 2 à 3 ans, éduqués dans les crèches n'était que 34.3\%, dont $58.9 \%$ dans les zones urbaines et seulement $8 \%$ dans les zones rurales» (Commission européenne, 2008, document non paginé).

On peut donc regrouper dans tous les cas les principales questions en trois grandes thématiques: modalités particulières de fonctionnement, performances scolaires et relations école/terriroire.

\section{Les trois âges de l'école rurale française}

L'école rurale a toujours suscité de nombreux débats. Malgré un évident recul lié aux évolutions sociales, économiques et démographiques, elle conserve aujourd'hui en France un poids important: avec près de $23 \%$ des élèves du primaire $^{1}$ et $19 \%$ des élèves de collège (11-16 ans), elle compte environ deux millions d'élèves, dont plus de 500000 dans le rural isolé. 
Le développement du système éducatif français depuis la fin du $19 \mathrm{e}$ siècle fait clairement apparaître trois grandes étapes, trois «âges» de l'école rurale, révélateurs des trois grandes questions qu'elle pose à la société française.

A la fin du $19^{\text {e }}$ siècle, l'enjeu est celui de l'unification nationale et de la modernité. L'école se voit confier une mission d'intégration sociale (Prost, 1992) pour des ruraux déjà perçus comme en marge ou à l'écart du progrès: il faut que leurs enfants abandonnent leurs "patois», qu'ils acquièrent les mêmes savoirs que les enfants des villes, pour pouvoir participer au grand mouvement de développement économique. L'école rurale apparaît alors, derrière sa fonction de socialisation (Durkheim, 1990) comme une machine à décentrer, qui doit faire disparaitre les particularismes, et remplacer les valeurs traditionnelles par des modèles plus favorables au "progrès». Elle constitue aussi le modèle de l'école, qui servira de référence - implicite ou explicite - pendant des décennies: elle représente l'image idéalisée de l'école publique, élément central de la vie de village et premier outil de l'ascension sociale des petits ruraux.

A partir de 1945, le très fort exode rural qui s'enclenche modifie complètement le contexte. La question de l'école devient celle du maintien d'un service public en milieu rural, dans le cadre des politiques d'aménagement du territoire, dont le but explicite est de s'occuper des exclus de la croissance, qui freinent par leur existence même l'élan général de la société française. La politique impulsée par la DATAR (Délégation à l'aménagement du territoire et à l'action régionale) est exemplaire à cet égard. Elle soutient les expériences et les innovations en milieu rural: équipes mobiles d'animation, réseaux d'écoles, usages des technologies de communication, etc. Il y a là une volonté explicite d'utiliser le système éducatif comme vecteur du changement socioculturel. Mais très vite vont apparaitre des exigences contradictoires: l'État s'oriente vers la rationalisation budgétaire et la recherche de l'efficience, tandis qu'au plan local la conception dominante est celle de l'école comme bien public assurant une mission fondamentale dont le coût doit être supporté par la solidarité nationale. Dans ces conditions, la question des performances scolaires va devenir centrale.

Les années soixante sont celles de la constitution en France d'un puissant courant de la sociologie de l'éducation, qui produit une «théorie de la reproduction» (Bourdieu \& Passeron, 1970). L'inégalité des chances scolaires entre dans le débat public, et pour l'école rurale, se trouve posée la question du «handicap»: les élèves ruraux seraient défavorisés à la fois par leur origine sociale et culturelle, (Alpe, 2006), par les spécificités du contexte territorial et par les conditions même de l'offre scolaire.

De très nombreuses publications vont tenter d'analyser, dans une perspective comparative, les performances scolaires des élèves ruraux. Les résultats, souvent contestés, ne font apparaître que bien peu de différences, et ne permettent en aucune façon de conclure à un «désavantage» manifeste et généralisé qui frapperait les élèves ruraux.

Dans le même temps, l'institution scolaire développe sa propre réponse, 
à travers la mise en place de dispositifs spécifiques, dont l'évaluation reste largement à faire, malgré les nombreux rapports de l'Inspection Générale de l'Éducation Nationale qui le reconnaissent (parfois) eux-mêmes (Duhamel, Houchot, Moulin \& Cuby, 2003). Ce mouvement est contemporain de la mise en place des IUFM (instituts universitaires de formation des maitres), qui s'accompagnera dans certains cas (Aix-Marseille, Auvergne, Grenoble, Lyon,...) de la création de modules de formation spécifique pour les futurs enseignants appelés à intervenir en zone rurale.

Il n'est évidemment pas possible de généraliser à partir de cette chronologie spécifiquement française, mais elle a le mérite de montrer comment la question de l'école rurale perdure en se transformant. Si les contextes institutionnels et les périodes different, les principales étapes (école rurale comme modèle de référence, prise en compte des effets de l'exode rural, émergence des questions de qualité de l'enseignement et d'égalité des chances) se retrouvent dans un grand nombre de pays.

\section{École rurale et «effet de territoire»}

Cette partie explore principalement la situation française, à partir d'enquêtes nationales et des résultats d'une recherche longitudinale (1997-2007) par suivi de cohorte sur un échantillon d'élèves ruraux français, menée dans le cadre de l'Observatoire de l'École Rurale ${ }^{2}$. 2400 élèves scolarisés en CM2 (dernière classe de l'école primaire) dans des écoles rurales de six départements français ${ }^{3}$ ont été interrogés en 1999 puis suivis jusqu'en Seconde (première classe du lycée) - pour les élèves n'ayant pas redoublé - à travers trois enquêtes réalisées en 2002, 2004 et 2005. Les parents ont aussi été interrogés.

La catégorie "école rurale» est définie par la commune où se trouve l'école, à travers la classification des communes françaises effectuée par l'Institut National des Statistiques et des Etudes Economiques (INSEE), qui décompose l'espace à dominante rurale (Champsaur, 1998) en quatre sous catégories: rural isolé, pôles ruraux, rural sous faible influence urbaine et rural sous forte influence urbaine cette dernière n'ayant pas été prise en compte dans l'enquête.

\section{Le débat sur les performances des élèves ruraux}

En France, ce débat est lancé dès 1963, avec les premières études sur l'entrée en sixième (première classe de l'enseignement secondaire) menées par l'Institut National d'Études Démographiques. À partir de là vont se succéder analyses et controverses, qui ont toutes pour objet de déterminer si les élèves ruraux sont défavorisés.

Les travaux menés par le ministère et coordonnés par Oeuvrard (2003), ainsi que les recherches de l'Observatoire de l'École Rurale (OER), (Alpe, Champollion $\&$ Poirey, 2003, 2005, 2006, 2010) permettent d'apporter des réponses claires. 
Tableau 1: Score des élèves ruraux et urbains* aux tests d'évaluation

\begin{tabular}{|l|l|l|}
\hline & Français & Mathématiques \\
\hline Ensemble des écoles rurales & 63,1 & 67,4 \\
\hline Ensemble des écoles urbaines & 63,2 & 67,3 \\
\hline
\end{tabular}

Source: Éducation et formation $N^{\circ} 43$, octobre 1995, Résultats nationaux, année scolaire 1993-1994

* élèves résidant dans des communes rurales ou urbaines au sens de l'INSEE

Cette première exploitation des résultats aux tests d'évaluation nationaux passés par tous les élèves lors de leur dernière année à l'école primaire (classe de CM2, soit 10-11 ans) est sans équivoque. Elle est corroborée par l'enquête de l'OER, portant sur les élèves ruraux de six départements français.

Tableau 2: Résultats des tests, évaluation Sixième*, 2000, base OER et résultats nationaux

\begin{tabular}{|l|l|l|l|}
\hline & & Français & Mathématiques \\
\hline & & \multicolumn{2}{|c|}{$\begin{array}{r}\text { Pourcentage d'élèves ayant obtenu } \\
\text { ce score }\end{array}$} \\
\hline Score $>90$ & OER & 8 & 10 \\
\hline & National & 5,8 & 8,1 \\
\hline Score $<30$ & OER & 1 & 3 \\
\hline & National & 2,4 & 5,9 \\
\hline
\end{tabular}

Source: Enquête OER, 1999 (2394 élèves) et Note d'information, MEN/DPD n01-36, juillet 2001 *première classe du collège, soit 11-12 ans

Les résultats des élèves ruraux sont globalement meilleurs, ce qui est d'autant plus remarquable que les origines sociales sont plus modestes. L'école rurale ne pénalise donc pas les élèves, du moins sur le plan des résultats scolaires au sortir de l'école: dans l'échantillon de l'OER (enquête 1999) ils sont d'ailleurs un peu moins en retard que la moyenne nationale.

Ce constat n'est pas complètement généralisable, car il ne concerne que des pays développés à haut niveau de vie: il est par exemple clairement vérifié par l'équipe du Groupement Interuniversitaire de l'École Rurale, Université de Barcelone (GIER) en Catalogne. Dans les pays européens les plus pauvres, l'école rurale est au contraire marquée par de très fortes inégalités, et les élèves ruraux ont les plus grosses difficultés à poursuivre leurs études au-delà de l'école primaire. En Roumanie par exemple, les résultats concernant la maitrise de la langue décrite comme "très bonne" passent de $70,8 \%$ en milieu urbain à $36 \%$ dans les écoles rurales (Birzea, 2002). Ces écoles connaissent aussi un déficit de compétences des enseignants (65\% d'enseignants non qualifiés contre $35 \%$ en ville), qui existe aussi, de façon beaucoup moins marquée, dans les pays plus riches. 
Mais les performances purement scolaires ne sont pas tout, et plusieurs recherches mettent l'accent sur la question des ambitions scolaires et professionnelles. Les souhaits d'orientation des élèves au sortir du collège (classe de Troisième: dernière classe du collège, soit 15-16 ans) en sont une très bonne illustration.

Tableau 3: Choix initiaux des élèves (premier choix) de $3 e^{*}$ et orientations effectives (en \%)

\begin{tabular}{|l|l|l|l|l|}
\hline & \multicolumn{2}{|l|}{ Collèges ruraux } & \multicolumn{2}{l|}{ Collèges urbains* } \\
\hline & Premier choix & $\begin{array}{l}\text { Orientation } \\
\text { effective }\end{array}$ & Premier choix & $\begin{array}{l}\text { Orientation } \\
\text { effective }\end{array}$ \\
\hline Seconde & 70,6 & 66,8 & 76,0 & 68,6 \\
\hline Redoublement & 3,1 & 9,1 & 3,3 & 12,8 \\
\hline BEP & 20,4 & 19,9 & 16,2 & 16,5 \\
\hline Apprentissage, CAP & 4,5 & 3,2 & 1,6 & 1,6 \\
\hline $\begin{array}{l}\text { Total Enseignement } \\
\text { professionnel court }\end{array}$ & 24.9 & 23,1 & 17,8 & 18,1 \\
\hline Autres & 1,4 & 1,0 & 2,9 & 0,5 \\
\hline
\end{tabular}

Source: Education et formation, $N^{\circ} 43$, octobre 1995

* la Troisième est la dernière classe du collège, l'orientation concerne l'entrée en Seconde (première classe du lycée)

** collèges situés dans des communes de plus de 10000 habitants

Les écarts constatés en faveur de l'enseignement professionnel court (CAP, $\mathrm{BEP}^{4}$, apprentissage) chez les élèves ruraux relèvent de deux ordres de fait:

- D'une part, ils portent sur eux-mêmes des jugements plus sévères, comme en témoigne ici les écarts entre les demandes de redoublement et les redoublements effectifs, trois fois plus nombreux pour les ruraux, près de quatre fois plus pour les urbains.

- D'autre part, les souhaits d'études longues sont plus rares, parce qu'un certain «réalisme» conduit les élèves ruraux à privilégier des débouchés professionnels présents dans leur environnement. Ces choix plus modestes mais plus réalistes sont mis en évidence dans des travaux du CEREQ (Arrighi, 2004) concernant l'insertion professionnelle des jeunes ruraux.

Reste une question centrale: comment expliquer ces différences? Les facteurs scolaires et culturels sont les plus souvent évoqués. 


\section{Les modalités de la socialisation scolaire} en milieu rural

\section{La petite taille des unités}

Cette caractéristique concerne à la fois le nombre de classes et leur effectif, et elle se vérifie pour les écoles comme pour les collèges. En effet, si les écoles à classe unique sont en voie de raréfaction (il en reste environ 5000 en France contre 19000 en 1960, et ce phénomène se retrouve partout, en Europe comme ailleurs), les écoles à deux ou trois classes sont encore très nombreuses en milieu rural.

Dans les collèges, la situation est plus contrastée. Au niveau national, les collèges situés dans l'espace à dominante rurale représentent $26,7 \%$ des collèges publics, mais seulement $18,2 \%$ des élèves, et le quart d'entre eux compte moins de 200 élèves. Ces faibles effectifs influent sur leur mode de fonctionnement et leur structure pédagogique.

Cette situation se retrouve dans les zones rurales de nombreux pays, avec parfois l'existence de sections professionnelles dès le collège (qui n'existent plus en France depuis plus de trente ans).

\section{L'isolement des enseignants}

Certains sont effectivement seuls (enseignants des écoles à classe unique), d'autres sont les seuls représentants de leur discipline (dans presque tous les petits collèges). Le contact avec les autres acteurs de l'institution scolaire (corps d'inspection, conseillers pédagogiques, formateurs, conseillers d'orientation,...) est plus rare et moins suivi. De plus, les personnels d'éducation autres que les enseignants sont souvent absents, ou affectés à temps partiel sur plusieurs établissements.

\section{L'offre éducative}

La diversité de l'offre scolaire (Oeuvrard, 2003) est beaucoup plus difficile à organiser dans des petites structures. Dans les écoles, c'est principalement le cas pour les intervenants extérieurs (en langues, en éducation physique, en informatique,...), puisque les maîtres du primaire sont censés enseigner toutes les disciplines scolaires. Dans les collèges, le nombre de langues vivantes proposées varie fortement selon la taille. $60 \%$ des collèges de moins de 100 élèves n'en proposent que deux, $33 \%$ en proposent trois, alors que dans les collèges de plus de 300 élèves, ces taux passent à $6 \%$ pour les deux langues et $64 \%$ pour les trois langues.

La réduction du nombre des formateurs rencontrés par un élève au cours de sa scolarité est un autre aspect du problème, plus difficile à analyser, mais sans doute lourd de conséquences. Les études sur les «effets-maître» (Felouzis, 1997) et "effets-établissements» (Cousin, 1998) ont souligné l'importance des effets séquentiels: la question n'est pas seulement de savoir si l'élève a un «bon maitre», il faut aussi tenir compte des moments de la scolarité (considérés comme plus 
ou moins décisifs - c'est le cas par exemple du moment de l'apprentissage de la lecture) et de la variété des conceptions pédagogiques des enseignants qu'il va rencontrer, et qui lui seront plus ou moins adaptées.

\section{L'environnement socioculturel}

L'équipement des petites structures rurales est un objet de préoccupations pour tous les acteurs locaux: parents, enseignants, élus. Certes, la présence des institutions culturelles (musées, théâtres, etc.) est nettement plus faible qu'en ville. A titre d'exemple, le pourcentage de communes équipées d'une bibliothèque passe de $91 \%$ dans les pôles urbains à $60,5 \%$ dans le rural isolé. De même, à l'intérieur des établissements, l'équipement informatique et audio-visuel est parfois plus difficile à obtenir, et il en est de même pour la présence et le fonctionnement des centres de documentation. Mais le fait le plus marquant est la très forte inégalité en la matière, à l'intérieur même du monde rural. En effet, la mobilisation des élus locaux est y plus qu'ailleurs décisive, et les moyens accordés par les communes aux écoles peuvent varier considérablement d'une petite école à l'autre: dans certaines zones rurales, l'écart est du simple au décuple. Ces moyens ont à la fois un effet interne (possibilité d'acheter des documents, du matériel) et externe (possibilités d'organiser des déplacements en ville,...) et ils influent bien évidemment sur le fonctionnement pédagogique. Cette grande variété des situations locales concerne aussi la présence et le dynamisme plus ou moins prononcé des structures culturelles locales. De ce fait, cette caractéristique du système éducatif en milieu rural doit être analysée au cas par cas, en fonction des réalités du terrain.

Il y a donc bien des caractéristiques objectives du système éducatif en milieu rural, qui distinguent clairement en France l'école rurale de l'école urbaine', et qui renvoient pour l'essentiel à des modalités spécifiques de la socialisation scolaire en milieu rural. Ceci explique pourquoi ces phénomènes, bien que facilement identifiables, soient difficiles à étudier quant à leurs effets sur la réussite scolaire des élèves: cela demande, en effet, que soit prise en compte la trajectoire scolaire de l'élève de son entrée à l'école jusqu'à sa sortie du collège c'est-à-dire le moment où, dans la quasi totalité des cas, il va intégrer un établissement urbain, puisque les lycées situés en zone rurale (même les lycées agricoles) sont extrêmement rares.

Très souvent, l'ensemble de ces particularités a été traité par les pouvoirs publics sous un seul angle, présenté comme principalement déterminant: l'isolement socioculturel ${ }^{6}$, qui constitue en fait un construit social, et non une réalité liée aux caractéristiques objectives des territoires.

\section{Isolement et "handicap culturel»}

La France possède, en matière d'éducation, une administration centrale particulièrement active. Dès qu'un «problème» est identifié, on voit se multiplier les études et les rapports. Parmi ceux-ci, ceux de l'Inspection Générale représentent 
pour le chercheur un ensemble de repères très intéressants sur le discours de l'institution.

En 1992, le Rapport Mauger (Agir ensemble pour l'école rurale, 1992) soulève un tollé dans le rural: au nom de l'efficacité et de l'équité, il préconise la suppression progressive de toutes les petites écoles pour faire de l'école à trois classes la norme de base: ainsi, avec une classe par cycle, tous les écoliers français auront "les mêmes chances». Les petits collèges (moins de 300 élèves $^{7}$ ) sont également visés, parce que les taux d'accès au lycée d'enseignement général y sont plus faibles qu'ailleurs. A partir de là, le raisonnement se développe: le Rapport Ferrier (consacré aux "réseaux scolaires en milieu rural», 1995) reprend la même idée et préconise, comme son nom l'indique, la mise en réseau des écoles. Le rapport Lebossé (Pour une nouvelle dynamique du système éducatif en zone rurale isolée, 1998) reprend une fois encore la même thématique, qui fera l'objet d'un texte officiel en 1999. Ce rapport reconnaît l'existence de résultats scolaires satisfaisants dans les petites écoles rurales, avec une réticence pour celles qui ont les plus petits effectifs, il entreprend une analyse critique de la politique préconisée depuis le Rapport Mauger (celle des «regroupements pédagogiques intercommunaux») et il introduit explicitement l'idée d'une "discrimination positive» (l'expression est citée en titre de paragraphe) pour l'école rurale. Mais c'est aussi celui où la stigmatisation du territoire transparaît le plus fortement.

Ainsi, le diagnostic est posé: isolement et petite taille vont de pair avec manque d'ouverture sur l'extérieur, absence d'activités culturelles ${ }^{8}$, demande d'éducation moins forte, et tout cela se traduira à terme par une orientation vers des études professionnelles courtes. Des affirmations comme celle concernant une demande sociale d'éducation moins forte en zone rurale témoignent d'ailleurs d'une totale ignorance des réalités du terrain: l'école rurale est, en France comme ailleurs, l'objet de mobilisations parfois très énergiques de la part de parents d'élèves, qui, rappelons-le, ressemblent de moins en moins à l'image des ruraux d'autrefois que semble avoir conservé l'institution scolaire.

De toute façon, ces rapports ne changent rien à l'essentiel: fermer les petites écoles, cela permet de récupérer des postes d'enseignants, et cela transfere aussi de l'État vers les collectivités territoriales une partie du coût de l'éducation. Car s'il est vrai que le coût par élève est plus faible dans de grosses structures, les coûts annexes, et en particulier celui du transport scolaire, augmentent très fortement, mais ce sont, en France, les départements qui payent ${ }^{9}$ !

La logique de l'institution est donc fort claire. Elle ne fait que reprendre la trilogie déjà évoquée dans les années 70 sous l'égide de la DATAR: regrouper, compenser, adapter... Mais les termes de cette injonction ne renvoient pas aux mêmes types de diagnostic et donc de mesures:

- Regrouper, c'est lutter contre l'isolement et la petite taille, et c'est très facile, il suffit de fermer les petits établissements, et de mettre en place des regroupements pédagogiques; 
- Compenser, c'est lutter contre le "handicap socioculturel» supposé peser sur l'école rurale: c'est le sens des politiques dites de «rupture d'isolement» dont il sera question ci-dessous;

- Par contre, adapter renvoie à une approche positive, qui supposerait que le système éducatif soit à même de se transformer en fonction de son contexte socioéconomique: les exemples existent, comme la «formation à la pluri activité en montagne» (Champollion, 1996) mais ils sont fort rares.

On voit donc clairement que, dans la façon dont l'institution analyse le système éducatif en milieu rural, le territoire pèse de tout son poids - mais il pèse uniquement comme un système de contrainte: isolement géographique et dispersion de la population sur des territoires peu peuplés, difficultés de communication, liées à la faiblesse des réseaux de toute nature et à l'isolement, pénurie de moyens matériels et humains, en relation avec les budgets restreints des collectivités locales, étroitesse de l'offre culturelle et périscolaire.

Le territoire n'est donc jamais perçu sous l'angle de la territorialité: la dynamique du système territorial est réduite à son expression la plus négative.

\section{Le territoire scolaire, objet de la territorialisation des politiques publiques}

\section{Les politiques de "rupture de l'isolement"}

Le traitement du "problème» des écoles rurales tente partout de répondre à un handicap supposé fondamental: celui de l'isolement. Il est indiscutable que celui-ci peut générer des difficultés sociales, organisationnelles et pédagogiques, mais celles-ci sont de fait des variables dépendantes d'un autre phénomène: celui des inégalités de moyens. Il existe dans les écoles rurales isolées des "riches» et des "pauvres» (souvent en fonction de la politique des collectivités territoriales de proximité), et l'origine sociale des élèves qui les fréquentent peut être aussi fortement contrastée. Plus généralement, l'isolement est un grave problème dans des pays pauvres où la géographie et la démographie représentent des contraintes difficilement dépassables (Afrique, pays andins, ...), il l'est beaucoup moins dans les pays développés, qui ont adopté partout le même type de solution: regroupement et réorganisation.

- Au Québec, «l'école éloignée en réseau» (Pévost, 2004) tente de pallier les énormes difficultés de distance et de transport, en s'appuyant sur des technologies de communication à distance et la mobilisation des communautés;

- En Catalogne, les "Zones d'écoles rurales» (ZER) ont la charge d'organiser les échanges entre écoles géographiquement voisines, de gérer un projet pédagogique commun et des services partagés d'enseignants affectés à la ZER elle-même et non à telle ou telle école. 
- En Italie, les regroupements autoritaires sont une pratique ancienne et durable, au point que dans certaines régions (Campanie) les petites écoles rurales ont presque complètement disparu.

En France, deux formes de regroupements pédagogiques intercommunaux (RPI) ont été expérimentées:

- Regroupements "éclatés», où des communes voisines se partagent les cycles scolaires: ce dispositif permet de garder une école dans chaque commune, et a des avantages pédagogiques évidents, mais génère énormément de déplacements des élèves;

- Regroupements concentrés, qui supposent la fermeture des écoles les plus petites au profit d'une école intercommunale. Ce modèle l'emporte très largement aujourd'hui (il concerne environ 10\% des élèves de l'école primaire française, mais le pourcentage est beaucoup plus élevé - parfois jusqu’à 100\% - dans les zones rurales).

Ils ont été accompagnés, dans les années 70 par des dispositifs innovants:

- Équipes mobiles académiques de liaison et d'animation (EMALA), constituées par des enseignants se déplaçant dans un réseau d'école avec un minibus et du matériel pédagogique, pour assister les enseignants isolés;

- «École rurale et Communication», qui rassemblait à intervalles réguliers enseignants et élèves d'écoles isolées pour entreprendre des activités en commun et regrouper temporairement les élèves par classe d'âge.

Dans tous les cas aussi, le recours aux technologies d'information et de communication a été privilégié. Cet engouement a sans doute eu des effets positifs sur l'ambiance générale des classes et des écoles et sur l'ouverture culturelle locale, mais il repose en partie sur une illusion: seuls ceux qui avaient déjà une politique d'ouverture et de communication active ont réellement bénéficié de ces technologies. Le seul avantage réel des écoles rurales en la matière résulte de leur structure: comme elles n'ont généralement pas de salle informatique, l'ordinateur est dans la classe, disponible à tout instant.

\section{Stigmatisation du territoire et politiques éducatives} La persistance d'un débat plus que séculaire sur l'école rurale ne peut rester sans effet sur les élèves.

Il est clair aujourd'hui que sur le plan strictement scolaire, les élèves ruraux ne sont pas spécialement défavorisés: les écarts de performances scolaires entre écoles à l'intérieur même des milieux ruraux et urbains sont beaucoup plus importants que les écarts globaux entre écoles rurales et écoles urbaines. Le «déficit culturel» des élèves ruraux n'existe pas non plus, sinon comme résultat d'une inégalité socio-économique inhérente à la répartition des catégories socioprofessionnelles sur le territoire (rappelons qu'aujourd'hui en France, le pourcentage d'ouvriers est plus important dans le rural que dans l'urbain). 
Pourtant, il existe des spécificités dans les parcours scolaires (voir tableau $\mathrm{n}^{\circ}$ 3 ), et les enquêtes de l'OER tendent à montrer qu'elles sont à mettre en relation avec la stigmatisation du territoire rural.

Les élèves ont parfaitement intégré au cours de leur scolarité et de leur adolescence les éléments de la représentation traditionnelle de l'école ou du collège rural. Alors qu'au CM2 (fin de l'école primaire, 10-11 ans), ils souhaitent majoritairement vivre et travailler à la campagne, leur préférence va à la petite ville en classe de Troisième (fin du collège, 14-15 ans), et leur répulsion envers la mobilité internationale, très forte au CM2, a disparu en Troisième, au profit de ce que nous avons appelé des "pays rêvés»: les pays limitrophes sont peu représentés et les USA représentent la destination la plus citée, sans que cela corresponde à des projets concrets - ce qui montre l'impact des cultures juvéniles et met une fois de plus à mal l'hypothèse simpliste de l'isolement culturel.

Les stéréotypes sont aussi largement présents dans l'idée qu'ils se font des avantages et inconvénients des collèges ruraux (voir tableau $\mathrm{n}^{\circ} 4$ ).

Tableau 4: Opinions des élèves ruraux (classes de Troisième) sur le collège rural* (Vocabulaire et locutions caractéristiques apparues au moins 30 fois)

\begin{tabular}{|l|c|l|c|}
\hline \multicolumn{2}{|l|}{ Avantage collège rural } & Inconvénient du collège rural \\
\hline & $\mathbf{N}^{* *}$ & & $\mathbf{N}^{*}$ \\
\hline Peu d'élèves & 418 & Eloignement & 122 \\
\hline Tranquillité & 138 & Trop petit & 91 \\
\hline Convivialité & 88 & Transports trop longs & 64 \\
\hline Campagne, nature & 65 & Peu de moyens & 64 \\
\hline Sécurité & 44 & Isolement & 52 \\
\hline Proximité & 37 & Moins bien équipé & 39 \\
\hline Tout le monde se connaît & 34 & Peu d'activités & 37 \\
\hline Peu d'élève par classe & 32 & Pas assez d'options & 30 \\
\hline
\end{tabular}

* collège situé dans une commune rurale au sens de l'INSEE

$N^{* *}$ : nombre d'occurrences - 1348 questionnaires - Source: Enquête OER, 2004

Les opinions des élèves sont souvent contradictoires (les mêmes éléments se retrouvent dans les deux colonnes) et parfois irréalistes ("transports trop longs», alors que les temps moyens sont souvent inférieurs aux temps de déplacement en zones urbaines), mais elles reflètent clairement l'image d'un "petit monde», plutôt convivial mais refermé sur lui-même, avec peu d'opportunités d'avenir.

Tout ceci alimente un pessimisme croissant: depuis les enquêtes de 1993, on sait que les projets des élèves ruraux sont souvent moins ambitieux que ceux des urbains (à niveau de performances scolaires identiques). Nos enquêtes le confirment: entre la fin de l'école primaire et la fin du collège, ils ont rabattu leurs ambitions. Ils envisagent des durées d'études plus courtes (le pourcentage 
de ceux qui désiraient suivre des études supérieures a diminué de moitié) et des métiers moins gratifiants (Grelet, 2006).

Dès lors, l'institution a beau jeu de se saisir de ces effets. Si les élèves ruraux sont moins ambitieux, il faut tout simplement les sortir du milieu rural. D'où les fermetures des petits établissements, les injonctions récentes pour développer à nouveau les internats permettant de regrouper les élèves à la ville et les «redéploiements» qui consistent en fait à réduire et concentrer les moyens.

Cela permet de répondre à une préoccupation purement gestionnaire, qui s'inscrit dans l'évolution récente des politiques éducatives, gagnées par le modèle entrepreneurial, portées par le développement d'une économie de l'éducation d'inspiration majoritairement libérale. Il faut réduire le "coût de l'élève», et il est clair qu'un élève rural dans un collège de 200 élèves coûte plus cher qu'un élève urbain dans une cité scolaire de 3000 élèves.

La volonté de "partir» (aller étudier à la ville, partir aux USA,...), fréquemment exprimée par les élèves ruraux sert aussi d'alibi et les politiques européennes de l'éducation favorisant la mobilité des étudiants sont souvent mises en avant par les politiques nationales: c'est «l'alibi de l'alibi». Mais cette volonté recouvre une réalité complexe:

- D'une part, elle est en contradiction avec les pratiques familiales, dans une Europe marquée par un vaste mouvement de "reruralisation», qui s'appuie à la fois sur des considérations économiques et des préoccupations environnementales;

- D'autre part, elle omet une caractéristique des stratégies socio-scolaires des élèves: nombreux sont ceux qui souhaitent partir pour revenir, leur détour par la ville étant inclus dans une stratégie réaliste de formation et leur projet de retour s'appuyant sur des considérations tout aussi réalistes sur les facilités de se loger et de trouver du travail dans un milieu que l'on maitrise à travers de nombreux réseaux sociaux.

- Enfin, ce n'est pas sans danger pour la société: «La seule voie ouverte aux enfants et aux jeunes provenant de familles pauvres et peu instruites souhaitant gravir l'échelle sociale reste l'émigration: la mobilité géographique comme facteur de mobilité sociale. Mais ce choix, tout à fait logique du point de vue de l'individu, conduit au vieillissement progressif de la population rurale, à l'appauvrissement et au déclin économique des espaces ruraux» (Commission européenne, 2008, document non paginé).

Nous assistons en fait à un retournement de l'histoire: l'école rurale, pensée il y a un siècle comme un vecteur de la modernité pour l'ensemble de la société, semble aujourd'hui devenue dans les pays riches un obstacle à la poursuite de la modernisation des comportements et des pratiques. Du fait que les politiques dites «territorialisées» le sont toujours par le haut (ce n'est jamais l'échelon local qui les inspire, mais l'administration centralisée qui les fait «descendre» vers les territoires ${ }^{10}$ ), les territoires sont invités à entrer dans le modèle dominant, sans 
que leurs spécificités soient prises en compte. Cette évolution est d'autant plus regrettable que l'école rurale, structure éducative de proximité, élément de la construction du lien social et de l'identité citoyenne, est à même de répondre à des questions brûlantes de nos sociétés. A l'heure où l'Europe se préoccupe de l'exclusion dans les zones rurales ${ }^{11}$ qui concerne tout particulièrement les nouveaux entrants, et de la "transition socioécologique» qui devrait marquer le $7^{\mathrm{e}}$ PCRDT $^{12}$, il parait absurde de se priver de cet atout.

\section{Conclusion}

L'école rurale a constitué - et constitue pour une part encore - le mythe fondateur d'une école républicaine: proposée à tous et partout, selon un idéal démocratique, elle a été concrètement le socle sur lequel s'est construite l'éducation moderne, et elle est restée, dans de nombreux pays (Angleterre, Canada, France, pays nordiques,...) un lieu d'expérimentation et d'innovation pédagogique, comme en témoigne par exemple la mise en place des dispositifs spécifiques évoqués ci-dessus - à tel point que l'on est parfois allée chercher dans l'école rurale les solutions aux difficultés rencontrées par les écoles urbaines de quartiers défavorisées. Dans le même temps, de nombreux discours l'ont accusée d'être une école de second rang, ne pouvant offrir à ses élèves les mêmes chances de réussite scolaire, au sens le plus académique du terme, alors que les enquêtes récentes montrent le contraire.

On peut dater la première crise de l'école rurale de la conjonction historique de deux phénomènes: l'exode rural et la massification scolaire (Duru-Bellat, 2006). En modifiant quantitativement et qualitativement à la fois la répartition de la population sur le territoire et la demande sociale d'éducation, elle a créé une situation de tension. La montée considérable des coûts de l'éducation, bien supportée par les budgets publics en temps de croissance économique, est devenue difficilement supportable en période de stagnation des recettes publiques.

De plus, les politiques de développement territorial qui s'étaient donné comme objectif de faire disparaître, à l'échelle des territoires nationaux les «exclus de la croissance» (zones rurales et de montagne, quartiers urbains défavorisées, zones en cours de désindustrialisation,...) ont connu globalement un affaiblissement spectaculaire ${ }^{13}$, et pour celles qui restent une réorientation (vers les technologies de communication par exemple).

Ainsi, la question de l'école rurale devient un révélateur des incohérences des politiques publiques d'éducation: axées sur la performance, pilotées sur un mode managérial, elles montrent une très faible capacité à résister à la marchandisation des services publics en général et des services d'éducation en particulier. Dans les pays les plus riches, on peut considérer le problème comme mineur, car il existe des compensations partielles (c'est le cas de la France, où l'enseignement secondaire accueille presque tous les élèves). Mais dans les pays ou les 
régions les plus pauvres, la présence du système éducatif en zone rurale reste une voie d'accès essentielle à la formation, et donc à l'insertion sociale, et constitue souvent le principal lieu de rencontre et d'animation culturelle. Il mérite donc d'être ardemment défendu.

\section{Notes}

1 En France, les élèves vont à l'école primaire de 6 à 11 ans, puis au collège (classes de la Sixième à la Troisième) de 11 à 15 ans, et ensuite au lycée (Seconde, Première, Terminale).

2 L'Observatoire de l'École rurale (OER) est une structure de recherche pluridisciplinaire (géographie, sciences de l'éducation, sociologie) créée en 1996 par des chercheurs et formateurs de cinq Instituts Universitaires de Formation des Maîtres (Aix-Marseille, Auvergne, Grenoble, Lyon, Franche-Comté. Ses membres sont principalement rattachés aujourd'hui à l'EA 4671 ADEF (Apprentissage, Didactique, Evaluation, Formation), Aix-Marseille Université / ENS Lyon - IFE

3 Ain, Alpes de Haute Provence, Ardèche, Drôme, Haute Loire, Haute Saône

4 CAP: certificat d'aptitude professionnelle, formation technique de 2 ans après la classe de Troisième. BEP: Brevet d'études professionnelles, supprimé en 2009 et intégré au baccalauréat professionnel.

5 Même si aujourd'hui certaines écoles urbaines ont introduit volontairement, pour des raisons pédagogiques, certaines de ces caractéristiques (les classes multi-âge en particulier) dans leur fonctionnement.

6 C'est aussi le cas en Catalogne: «Un des principaux problèmes auquel a dû, historiquement, faire face l'école rurale catalane (et aussi espagnole) [...] a été l'isolement», (GIER, 2006)

7 Rappelons qu'à la rentrée 2003, il y avait encore 350 collèges publics de moins de 200 élèves.

8 Ce qui évidemment tout à fait faux. Sur ce point, voir Alpe, 2006.

9 En France, les communes ont la charge matérielle de la construction et de l'entretien des écoles, les départements celle des collèges et des transports scolaires (hors salaires). La part des collectivités territoriales dans le financement de la dépense intérieure d'éducation atteint aujourd'hui 23,6 \% (Repères et références statistiques, Ministère de l'Éducation Nationale, 2012). En Roumanie, lors de l'étude menée pour le Ministère en 2001-2002, les responsables de l'administration centrale n'ont eu de cesse de dénoncer la faible implication financière des communes rurales dans l'entretien et l'équipement de leurs écoles - moyen efficace de dédouaner l'État de ses responsabilités (Alpe, 2002).

10 En France, la réforme en cours de la représentation élective des territoires (diminution drastique du nombre des représentants élus et création de "conseillers territoriaux» élus sur des scrutins de liste, donc sans base territoriale) est un autre exemple de cette tendance.

11 Commission européenne (2008). Pauvreté et exclusion dans les zones rurales, Direction générale de l'emploi, des affaires sociales et de l'égalité des chances, Unité E2. 
12 PCRDT: Programme 2007-2013, Cadre de Recherche et Développement Technologique de la Commission européenne qui finance la coopération en matière de recherche.

13 En France, effacement de la DATAR (délégation à l'aménagement du territoire et à l'action régionale), en Europe disparition annoncée des programmes LEADER pour les pays les plus avancés.

\section{Références bibliographiques}

Alpe, Y. (2002). Romanian rural education project. Rapport de mission pour la Banque mondiale. Bucarest: Ministry of Education of Romania / World Bank.

Alpe, Y. (2006). Existe-t-il un «déficit culturel» chez les élèves ruraux? Revue Française de Pédagogie, 156, 75-88.

Alpe, Y., Champollion, P. \& Poirey, J. L. (Éd.). (2003). L'enseignement scolaire dans les milieux ruraux et montagnards (Tome 2: Au seuil du collège). Besançon: Presses Universitaires Franc-comtoises.

Alpe, Y., Champollion, P. \& Poirey, J. L. (Éd.). (2004). L'enseignement scolaire dans les milieux ruraux et montagnards (Tome 3: Collégiens à mi-parcours). Besançon: Presses Universitaires Franc-comtoises.

Alpe, Y., Champollion, P. \& Poirey, J. L. (Éd.). (2006). L'enseignement scolaire dans les milieux ruraux et montagnards (Tome 4: Le devenir des élèves en fin de collège: Parcours et projets). Besançon: Presses Universitaires Franc-comtoises.

Alpe, Y., Champollion, P. \& Poirey, J. L. (Éd.). (2010). L'enseignement scolaire dans les milieux ruraux et montagnards (Tome 5: Après le collège). Besançon: Presses Universitaires Franc-comtoises.

Alpe, Y. \& Fauguet, J. L. (2008). Sociologie de l'école rurale. Paris: L'Harmattan.

Arrighi, J. J. (2004). Les jeunes dans l'espace rural: Une entrée précoce sur le marché du travail ou une migration probable. Formation-Emploi, 87, 63-78.

Birzea, C. (2002). The current state of rural education in Romania. Rapport pour le Ministère de l'Éducation de Roumanie [non publié]. Bucarest: Ministère de l'Éducation.

Carrier, M. \& Beaulieu, P. (1995). Les petites écoles primaires en milieu rural au Québec: Fermeture, maintien ou développement? Consulté le 10 novembre 2010 dans http://web2. uqat.ca/ chairedesjardins/documents/Carrier_Petites_ecoles_1995.PubROCR.pdf

Champollion, P. (1996). La formation en montagne: État des lieux, problématique et enjeux. Actes du congrès de la Fédération Française d'Économie Montagnarde "Pour une relance de la politique montagne: La formation, un atout», Saint-Jean-la-Vêtre (F).

Champsaur, P. (Éd.). (1998). Les campagnes et leurs villes. Paris: INSEE.

Commission européenne. (2008). Pauvreté et exclusion sociale dans les zones rurales. Direction générale de l'emploi, des affaires sociales et de l'égalité des chances.

Cousin, O. (1998). L'efficacité des collèges. Sociologie de l'effet établissement. Paris: PUF.

Dauthuile, P. (1900). L'école primaire dans les Basses-Alpes, depuis la Révolution jusqu'à nos jours. Digne: Vial.

Duhamel, M., Houchot, A., Moulin, Y. \& Cuby, J.-F. (2003). L'évolution du réseau des écoles primaires: Rapport définitif (Inspection générale de l'éducation nationale, Inspection générale de l'administration de l'éducation nationale et de la recherche, $\mathrm{N}^{\circ}$ 03-028 et 03-048). Paris: Bibliothèque des rapports publics, La Documentation Française.

Durkheim, E. (1990). Éducation et sociologie. Paris: PUF Quadrige. (Original publié1922)

Duru-Bellat, M. (2006). L'inflation scolaire. Paris: Seuil.

Felouzis, G. (1997). L'efficacité des enseignants: Sociologie de la relation pédagogique. Paris: PUF.

GIER. (2006). L'école rurale en Catalogne: Une vision d'ensemble dans une perspective multidisciplinaire. In Y. Alpe, P. Champollion \& J. L. Poirey (Éd.), L'enseignement scolaire 
dans les milieux ruraux et montagnards (Tome 4: Le devenir des élèves en fin de collège: Parcours et projets, pp. 167-186). Besançon: Presses Universitaires Franc-comtoises.

Grelet, Y. (2006). Des territoires qui façonnent les parcours scolaires des jeunes. Bref, 228, $1-4$.

Lebossé, J.-C. (1998). Pour une nouvelle dynamique du système éducatif en zone rurale isolée. Rapport au Ministère de l'Éducation Nationale. Paris: Bibliothèque des rapports publics, La Documentation Française.

Monjo, R. (1998). La «forme scolaire» dans l'épistémologie des sciences de l'éducation. Revue française de Pédagogie, 125, 83-93.

Oeuvrard, F. (2003). Les performances de l'école rurale, quelle mesure, dans quel objectif? Ville-École Intégration Enjeux, 134, 151-164.

Pévost, P. (2004). Projet L'école éloignée en réseau. Les collaborations école-communauté au Québec: Une perspective de développement local au moyen de quatre études de cas (Rapport de recherche). Québec: Université de Sherbrooke. Consulté le 12 octobre 2010 dans http:// www.cefrio.qc.ca/projets/proj_34.cfm

Prost, A. (1992). Education, société et politiques. Une histoire de l'enseignement en France de 1945 à nos jours. Paris: Seuil.

Vincent, G. (1994). L'Éducation prisonnière de la forme scolaire? Scolarisation et socialisation dans les sociétés industrielles. Presses Universitaires de Lyon.

Mots clés: École rurale, performances scolaires, politiques éducatives, territoire

\section{Bildungswesen im territorialen Geltungsbereich: die ländliche Schule in Frankreich - von der Idealisierung zur Stigmatisierung}

\section{Zusammenfassung}

Das Bildungssystem im ländlichen Raum ist zeit seines Bestehens von Spannungen durchzogen; aktuell stattfindende sozio-ökonomische Entwicklungen verschärfen diese Spannungen noch zusätzlich. Oft steht die Sozialisierungsfunktion der ländlichen Schule im Vordergrund, ebenso häufig erforscht werden aber die Schulleistungen von Lernenden ländlicher Schulen, welche als geringer angenommen werden als die Leistungen derjenigen mit städtischer Herkunft. Dabei wurde zum einen die Frage der Stigmatisierung von ländlichen Gebieten und deren Wirkungen auf die Akteure im Bildungswesen gestellt, und zum andern die Frage der raumbezogenen Bildungspolitiken. Hier bestehen aber oft Diskrepanzen zu den Forschungsergebnissen, wie das hier entwickelte Beispiel Frankreich zeigt.

Schlagworte: Ländliche Schule, Schulleistungen, Bildungspolitiken, Territorium 


\section{Contesto territoriale e organizzazione scolastica: la scuola rurale francese, dall'idealizzazione alla stigmatizzazione}

\section{Riassunto}

Il sistema educativo nel contesto rurale è sin dalle sue origini attraversato da tensioni, aggravate dall'evoluzione recente del contesto socio-economico del mondo rurale. La funzione specifica della scuola rurale nel processo di socializzazione è stata spesso messa in risalto, e le prestazioni scolastiche degli allievi rurali, supposte inferiori a quelle degli allievi in contesto urbano, sono state oggetto di numerosi studi che invitano a riflettere, da una parte, sul problema della stigmatizzazione dei territori rurali e dei relativi effetti sugli attori scolastici, e che, in secondo luogo, delineano la questione delle politiche educative territorializzate, spesso sfasate rispetto ai risultati della ricerca, come mostrato dall'esempio della Francia qui proposto.

Parole chiave: Scuola rurale, prestazioni scolastiche, politiche educative, territorio.

\section{Territorial context and school organization: French rural school, from idealization to stigmatization}

\section{Summary}

The education system in rural areas has always had problems. These have been aggravated recently by the socio-economic climate. The function of rural schools as a place for pupils to socialise is widely acknowledged, and celebrated. In contrast, the academic performance of rural schools students is often reputed to be inferior to that of their urban counterparts - an assumption that has been the subject matter of numerous studies. These studies question whether the stigmatisation of rural areas affects the attitude of classroom staff towards pupils, and the part played by differences in territorial education policies in creating disparages in academic performance, as shown in the French study.

Key words: Rural school, academic performance, education policies, territories 
